\title{
Poliquetos (Annelida: Polychaeta) asociados a algas rojas intermareales de Córdoba, Caribe Colombiano
}

\author{
Polychaetes (Annelida: Polychaeta) associated with intertidal \\ red algae of Córdoba, Colombian Caribbean
}

\section{Jorge Quirós-Rodríguez ${ }^{1}$, Pedro Dueñas Ramírez² y Néstor Hernando Campos ${ }^{3}$}

\author{
${ }^{1}$ Departamento de Biología. Facultad de Ciencias Básicas, Universidad de Córdoba, Carrera 6a No. 76-103, Montería, \\ Córdoba, Colombia. alexander_quiroz@hotmail.com \\ ${ }^{2}$ Facultad de Ciencias Naturales, programa de Biología Marina, Universidad Jorge Tadeo Lozano, Santa Marta, Colombia. \\ pedror.duenasr@utadeo.edu.co \\ ${ }^{3}$ Centro de Estudios en Ciencias del Mar CECIMAR, Universidad Nacional de Colombia, Sede Caribe, Santa Marta, Colombia. \\ nhcamposc@unal.edu.co
}

\begin{abstract}
Between September 2006 and June 2007 the composition and abundance of polychaetes associated with intertidal red algae was studied, in 7 stations along the coast of Córdoba, Colombian Caribbean. To collect the specimens a quadrant of $625 \mathrm{~cm}^{2}$ with 5 replicates randomly arrange at each sampling point was used. All intertidal red algae fronds examined contained polychaetes with a record of 288 individuals belonging to 19 species of 7 families. The most abundant species was Platynereis dumerilii (Nereididae) with 188 individuals, followed by Pseudonereis gallapagensis (Nereididae) with 23 individuals and Syllis corallicola (Syllidae) with 12 individuals. Also the sedentary polychaetes, Hydroides sanctaecrusis (Serpulidae) with 4 individuals and Pista palmata (Terebellidae) with 5 individuals were collected. The highest abundance of polychaetes abundance was recorded in December (T2) and March (T3) with 79 and 103 individuals respectively, and the lowest in September (T1) and June (T4) with 47 and 59 individuals. According to the ANOSIM test there were no significant differences among groups $(\mathrm{R}=0.09, P=0.52)$. This is because the polychaete $P$. dumerilii had the highest similarity contribution (> 77\%) in each group, indicating which was the most important in terms of abundance in all sampling months. Finally, the species collected in the algal fronds are characteristic species of soft bottom and rocky substrates, suggesting a non-specific adaptation of these species to substrates algal.
\end{abstract}

Key words: Platynereis dumerilii, Rhodophyta, communities, coastline, Córdoba

\begin{abstract}
Resumen.- Entre septiembre 2006 y junio 2007 se estudió la composición y abundancia de poliquetos asociados a algas rojas intermareales, en 7 estaciones de la franja costera cordobesa del Caribe colombiano. Para la recolección de los especímenes, se delimitó un cuadrante de $625 \mathrm{~cm}^{2}$, con 5 réplicas dispuestas al azar en cada punto de muestreo. Todos los frondes de algas rojas examinados evidenciaron la presencia de poliquetos, con un registro de 288 individuos pertenecientes a 19 especies de 7 familias. La especie más abundante fue Platynereis dumerilii (Nereididae) con 188 individuos, seguida de Pseudonereis gallapagensis (Nereididae) con 23 individuos y Syllis corallicola (Syllidae) con 12 individuos. También se colectaron los poliquetos sedentarios, Hydroides sanctaecrusis (Serpulidae) con 4 individuos $y$ Pista palmata (Terebellidae) con 5 individuos. La abundancia más alta de poliquetos se registró en diciembre (T2) y marzo (T3) con 79 y 103 individuos respectivamente, y la menor ocurrió en septiembre (T1) y junio (T4) con 47 y 59 individuos. Con relación a la prueba ANOSIM, no se registró una agrupación significativamente diferente de la otra $(R=0,09, P=0,52)$, debido a que $P$. dumerilii mostró el más alto porcentaje de contribución (> 77\%) en cada agrupación, lo que indica que fue la especie más importante, en términos de abundancia, durante los meses de muestreo. Finalmente, las especies colectadas en los frondes algales son especies características de sustratos de fondos blandos y rocosos, sugiriendo una adaptación no específica de estas especies a los sustratos algales.
\end{abstract}

Palabras clave: Platynereis dumerilii, rodófitas, comunidades, franja costera, Córdoba

\section{INTRODUCCIÓN}

Entre los ambientes marinos litorales, los frondes algales constituyen un sustrato favorable para el asentamiento de epibiontes (Winston \& Eiseman 1980, Seed \& O’Connor 1981), de esta forma los poliquetos seleccionan el alga facilitadora, basándose tanto en características internas como externas, aunque la forma (o complejidad estructural) del alga es un regulador más fuerte que la palatabilidad o las defensas químicas (Bates 2009). La 
cobertura y longitud de los frondes son también, consideradas para evaluar la complejidad estructural de las macroalgas (Jenkins et al. 2002, Kelaher \& Castilla 2005) y en general, una mayor complejidad estructural de los frondes implica una mayor biodiversidad de las especies asociadas (Bates \& De Wreede 2007, Unsworth et al. 2007, Bates 2009). No obstante, esta relación no es lineal, ya que existe un umbral de complejidad por encima del cual se origina una relación inversa entre la estructura del hábitat y la biodiversidad (Kelaher \& Castilla 2005).

Entre las macroalgas de mayor cobertura y rango de distribución para el Caribe colombiano, se encuentran las algas rojas, resaltándose Gracilaria mammillaris (Montagne) M.A. Howe, 1918 (Gracilariaceae), G. damaecornis J. Agardh, 1852, G. cervicornis (Turner) J. Agardh, 1852, Bryothamnion triquetrum (S.G. Gmelin) M.A. Howe, 1915 (Rhodomelaceae) y B. seaforthii (Turner) Kützing, 1843, adheridas principalmente a corales, rocas y sustratos duros de la zona intermareal (García \& Díaz-Pulido 2006, Quirós-Rodríguez et al. 2010). Por esta razón, el conocimiento de los ensambles de poliquetos en los frondes de las algas rojas intermareales, es considerado importante para caracterizar los distintos hábitats bentónicos (Alós et al. 1982) y fundamental en programas de monitoreo ambiental (Cañete et al. 2000).

Existen estudios sobre poliquetos asociados en agregados de ascidias (Zamorano \& Moreno 1975), cirripedios (Hernández et al. 2001), bivalvos (Paredes \& Tarazona 1980, Liñero-Arana \& Díaz-Díaz 2006) y agregaciones en tubos de otros poliquetos (Sepúlveda et al. 2003). En las costas de Chile se destacan los estudios de biodiversidad de poliquetos en sustratos algales, principalmente en las algas pardas Lessonia nigrescens Bory, 1826 (Vásquez \& Santelices 1984, Vásquez \& Vega 2005) y Macrocystis pyrifera (Linnaeus) C. Agardh, 1820 (Cariceo et al. 2002, Ríos et al. 2007, Ríos \& Mutschke 2009), así como en el alga roja calcárea Corallina officinalis Linnaeus, 1758 (López \& Stotz 1997, Kelaher 2002).

Actualmente, en el Caribe colombiano no existen registros acerca de poliquetos asociados a los frondes algales, por lo que esta investigación aporta significativamente al conocimiento de la diversidad de poliquetos, y el efecto de los frondes algales en estos organismos. El objetivo del estudio fue determinar la composición y abundancia de poliquetos asociados a las algas rojas intermareales de Caribe cordobés.

\section{Materiales y MÉTOdos}

\section{ÁREA DE ESTUDIO}

El estudio se realizó en el litoral Caribe cordobés, entre $9^{\circ} 25^{\prime} \mathrm{N}$ y $75^{\circ} 42^{\prime} \mathrm{W}$ en el corregimiento de El Porvenir y $8^{\circ} 54^{\prime} \mathrm{N}$ y $76^{\circ} 26^{\prime} \mathrm{W}$ en punta Arboletes (Fig. 1). El litoral costero cordobés corresponde a una zona de bosque seco tropical, según el patrón de zonación de Höldridge (1978), presentando una época seca (diciembre a marzo) y una época lluviosa (abril a noviembre). En julio se presenta una disminución de las lluvias, el veranillo de San Juan. La extensión del litoral cordobés con 140 km, está caracterizada por la influencia de la actividad antrópica, el aporte de aguas continentales, la geomorfología costera y el nivel de energía del oleaje, dividiendo la zona en 2 ecorregiones (Tabla 1), de acuerdo con lo establecido por INVEMAR (2000).

\section{ECORREGIÓN MoRrosquillo}

Comprende la región desde punta Barú hasta la desembocadura del río Sinú, recibiendo la descarga continental de este río, la del canal del Dique y la de otros ríos de menor caudal (Ardila et al. 2002). La energía del oleaje es predominantemente baja, debido a que los cuerpos de agua son semicerrados, e.g., golfo de Morrosquillo y bahía de Cispatá. Los sedimentos de la zona son de textura fina, en su mayoría, y se observan manglares desarrollados en los diversos sistemas lagunoestuarinos. Las praderas de fanerógamas son características de la región (INVEMAR 2000). El Morrosquillo cordobés se caracteriza por comprender una extensa llanura costera con terrazas coralinas, situación que permitió la formación de parches rocosos arrecifales muertos a lo largo de la franja costera y el desarrollo de asociaciones multiespecíficas de algas filamentosas (Quirós-Rodríguez et al. 2010).

\section{ECORREGIÓN DARIÉN}

Esta región se localiza desde la desembocadura del río Sinú hasta cabo Tiburón, incluyendo el golfo de Urabá (Ardila et al. 2002). Se encuentra altamente influenciada por las descargas continentales de los ríos Sinú, Atrato y otros de menor caudal, lo que divide la zona en 3 partes. Está constituida por fondos lodosos en su gran mayoría y una plataforma continental relativamente ancha. La primera subregión comprende el Darién cordobés, entre el río Sinú y Punta Arboletes, con una costa baja, erosiva y conformada predominantemente por playas y 


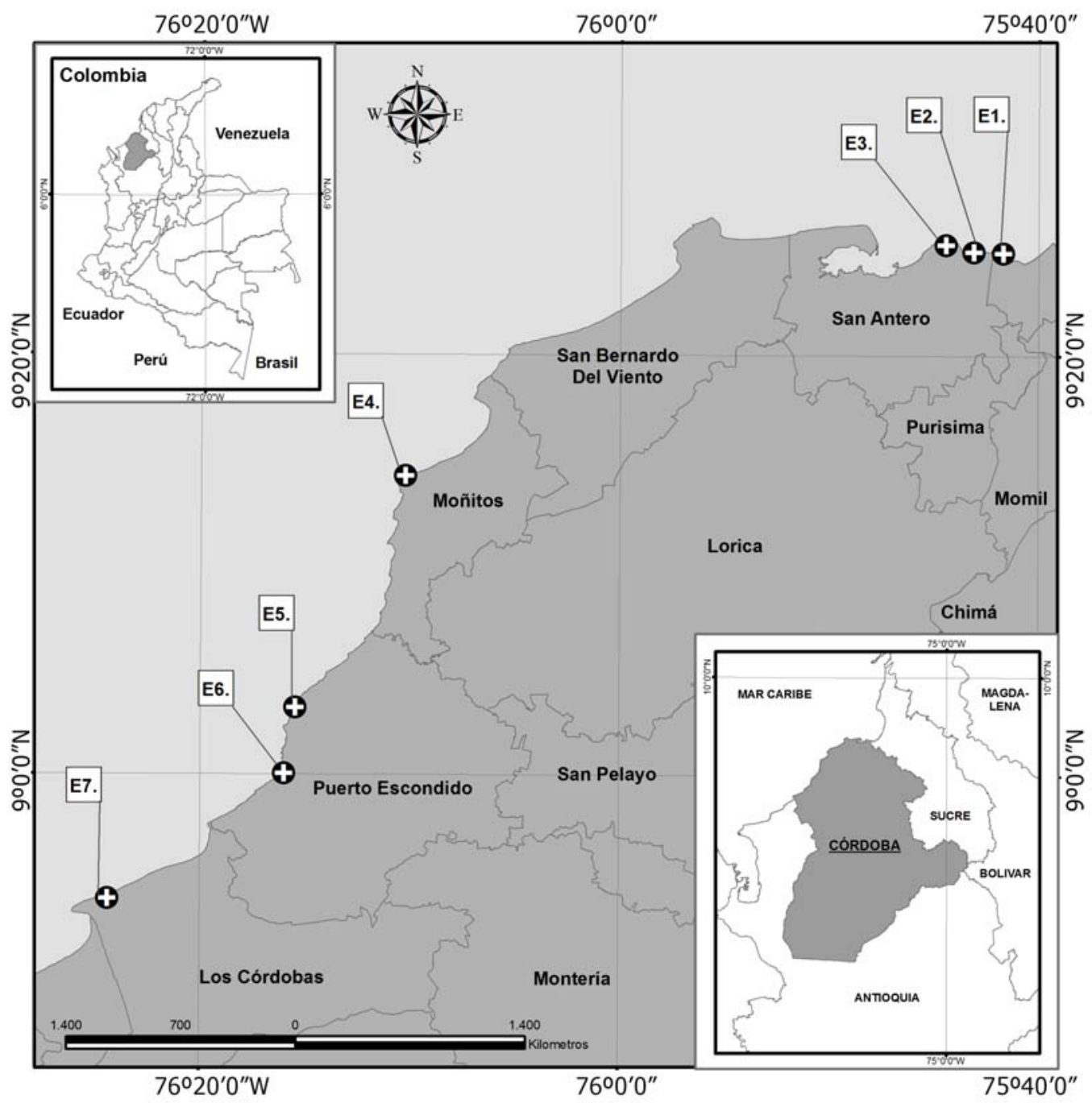

Figura 1. Localización del área de estudio y estaciones de muestreo en la franja costera del departamento de Córdoba / Location of the study area and sampling stations along the rocky coastline of Córdoba

Tabla 1. Ubicación geográfica y tipo de sustrato en las 7 estaciones, Córdoba, Caribe Colombiano / Geographic location and type of substrate in the 7 stations, Córdoba, Colombian Caribbean

\begin{tabular}{lccccc}
\hline Puntos de muestreo & Ecorregión & Localidad & Latitud & Longitud & Tipo de sustrato \\
\hline Estación 1 & Morrosquillo & San Antero & $9^{\circ} 25.016 \mathrm{~N}$ & $75^{\circ} 42.235 \mathrm{O}$ & Rocoso calcáreo \\
Estación 2 & Morrosquillo & San Antero & $9^{\circ} 25.037 \mathrm{~N}$ & $75^{\circ} 42.251 \mathrm{O}$ & Rocoso calcáreo \\
Estación 3 & Morrosquillo & San Antero & $9^{\circ} 25.135 \mathrm{~N}$ & $75^{\circ} 42.857 \mathrm{O}$ & Rocoso calcáreo \\
Estación 4 & Darién & Moñitos & $9^{\circ} 13.095 \mathrm{~N}$ & $76^{\circ} 10.068 \mathrm{O}$ & Rocoso calcáreo \\
Estación 5 & Darién & Puerto Escondido & $9^{\circ} 03.201 \mathrm{~N}$ & $76^{\circ} 15.502 \mathrm{O}$ & Rocoso sedimentario \\
Estación 6 & Darién & Puerto Escondido & $9^{\circ} 00.460 \mathrm{~N}$ & $76^{\circ} 15.544 \mathrm{O}$ & Rocoso sedimentario \\
Estación 7 & Darién & Los Córdobas & $8^{\circ} 53.735 \mathrm{~N}$ & $76^{\circ} 24.580 \mathrm{O}$ & Rocoso artificial \\
\hline
\end{tabular}


Tabla 2. Listado taxonómico de algas rojas intermareales de Córdoba / Taxonomic list of Intertidal red algae of Córdoba

\begin{tabular}{|c|c|c|c|c|c|c|c|}
\hline & \multicolumn{3}{|c|}{$\begin{array}{l}\text { Ecorregión } \\
\text { Morrosquillo }\end{array}$} & \multicolumn{4}{|c|}{ Ecorregión Darién } \\
\hline & Est.1 & Est.2 & Est.3 & Est.4 & Est.5 & Est.6 & Est.7 \\
\hline \multicolumn{8}{|l|}{ RHODOPHYTA } \\
\hline \multicolumn{8}{|l|}{ Ceramiaceae } \\
\hline Ceramium cimbricum H.E. Petersen, 1924 & $*$ & * & $*$ & * & $*$ & * & $*$ \\
\hline Centroceras clavulatum (C. Agardh) Montagne, 1846 & & * & $*$ & * & & & * \\
\hline \multicolumn{8}{|l|}{ Gelidiaceae } \\
\hline Gelidium pusillum (Stackhouse) Le Jolis, 1863 & * & * & $*$ & $*$ & $*$ & $*$ & $*$ \\
\hline \multicolumn{8}{|l|}{ Hypneaceae } \\
\hline Hypnea musciformis (Wulfen) J.V Lamouroux, 1813 & * & * & $*$ & * & $*$ & * & $*$ \\
\hline Hypnea spinella (C.Agardh) Kützing, 1847 & & & * & $*$ & $*$ & & * \\
\hline \multicolumn{8}{|l|}{ Gelidiellaceae } \\
\hline Gelidiella acerosa (Forsskål) Feldmann \& G. Hamel, 1934 & * & * & $*$ & * & & * & \\
\hline \multicolumn{8}{|l|}{ Halymeniaceae } \\
\hline Grateloupia filicina (J.V.Lamouroux) C. Agardh, 1822 & & & & * & & * & $*$ \\
\hline Halymenia elongata C.Agardh, 1822 & & & & * & & $*$ & \\
\hline \multicolumn{8}{|l|}{ Rhodomelaceae } \\
\hline Acanthophora muscoides (Linnaeus) Bory de Saint-Vincent, 1828 & * & * & * & & & & \\
\hline Acanthophora spicifera (M.Vahl) Børgesen, 1910 & * & * & * & & & & \\
\hline Laurencia obtusa (Hudson) J.V. Lamouroux, 1813 & * & & * & * & $*$ & * & \\
\hline Palisada perforata (Bory de Saint-Vincent) K.W.Nam, 2007 & * & * & $*$ & * & * & * & \\
\hline Bryothamnion triquetrum (S.G. Gmelin) M.A.Howe, 1915 & & * & * & * & * & * & \\
\hline Bryothamnion seaforthii (Turner) Kützing, 1843 & * & * & $*$ & * & $*$ & * & \\
\hline Bostrychia moritziana (Sonder ex Kützing) J. Agardh, 1863 & & & & * & $*$ & * & * \\
\hline \multicolumn{8}{|l|}{ Solieriaceae } \\
\hline Solieria filiformis (Kützing) P.W. Gabrielson, 1985 & * & * & * & * & & & \\
\hline \multicolumn{8}{|l|}{ Galaxauraceae } \\
\hline Dichotomaria obtusata (J. Ellis \& Solander) Lamarck, 1816 & & * & $*$ & & & & \\
\hline \multicolumn{8}{|l|}{ Gracilariaceae } \\
\hline Gracilariopsis lemaneiformis (Bory) Dawson, Acleto \& Fodvik, 1964 & * & $*$ & & & & & \\
\hline Gracilaria mammillaris (Montagne) M.A. Howe, 1918 & * & $*$ & $*$ & $*$ & $*$ & * & $*$ \\
\hline Gracilaria domingensis (Kützing) Sonder ex Dickie, 1874 & & & & * & $*$ & * & \\
\hline Hydropuntia caudata (J.Agardh) Gurgel \& Frederick, 2004 & * & * & & & & & \\
\hline Gracilaria cervicornis (Turner) J. Agardh, 1852 & * & * & $*$ & & & & \\
\hline Gracilaria damaecornis J. Agardh, 1852 & * & $*$ & $*$ & & & & $*$ \\
\hline Gracilaria blodgettii Harvey, 1853 & $*$ & & & $*$ & $*$ & * & $*$ \\
\hline Total de especies & 16 & 17 & 17 & 17 & 12 & 14 & 10 \\
\hline
\end{tabular}

acantilados bajos, de material poco cohesivo. Las aguas son turbias cerca de la costa, pero se tornan transparentes en áreas de mayor profundidad. Los manglares, corales y praderas de fanerógamas son poco representados en este sitio (INVEMAR 2000, Quirós-Rodríguez et al. 2010).

\section{DiSEÑo DE MUESTREO}

Se realizaron muestreos trimestrales entre septiembre/06 y junio/07, con el fin de abarcar 2 periodos climáticos del año (época seca y época lluviosa). Se recolectó material en 7 estaciones, 3 se ubicaron en la ecorregión Morrosquillo y 4 en Darién, cubriendo sustrato rocoso calcáreo, sedimentario y artificial. En cada punto de muestreo se ubicó un cuadrante de $625 \mathrm{~cm}^{2}$ y se realizaron 5 réplicas. En todos los cuadrantes se evaluó la cobertura de las algas rojas a nivel específico. Para la separación de las algas rojas desde su disco de fijación en el sustrato, se empleó una espátula metálica, siendo inmediatamente 
colocadas en recipientes plásticos realizando una primera separación de los poliquetos adheridos a los frondes algales. Posteriormente, se filtró y lavó el contenido algal con un tamiz de 250 y $500 \mu \mathrm{m}$ y se recogieron los poliquetos, los cuales fueron extraídos y preservados en formalina al $10 \%$.

\section{IDENTIFICACIÓN DEL MATERIAL}

La identificación taxonómica de las algas colectadas, se realizó con base en los trabajos de Taylor (1975), Schnetter (1980), Bula-Meyer (1998) y Littler \& Littler (2000). Para la corroboración de las muestras identificadas en el laboratorio, se contó con la asesoría del grupo de Investigación en Ecología de la Universidad del Magdalena y el grupo de Investigación en Biodiversidad de la Universidad de Córdoba. En la identificación taxonómica de los poliquetos colectados en este estudio, se realizó con base en los trabajos de Hartmann-Schröder (1962, 1965), Fauchald (1977) y Rozbaczylo (1980).

\section{OBTENCIÓN Y TRATAMIENTO DE LOS DATOS}

Para determinar la estructura de las algas rojas, se estimó la riqueza total (S) y la abundancia como porcentaje de cobertura (\%) en cada una de las estaciones de muestreo. La estructura de los poliquetos se estimó a través de la riqueza total (S) y número total de individuos $(\mathrm{N})$ por estación. Así mismo, para determinar la afinidad entre las muestras, se utilizó la técnica de ligamiento promedio no ponderada (UPGMA) y el escalamiento multidimensional no métrico (NMDS), con las abundancias de las especies de poliquetos transformadas a raíz cuarta, utilizando la matriz de similaridad de Bray \& Curtis (1957). El análisis de similaridad (ANOSIM), se realizó para identificar si hay diferencias significativas entre agrupaciones (meses de muestreo), teniendo como factor las estaciones (E1E7). Además, se estimó el análisis de similitud porcentual (SIMPER), para evaluar el grado de contribución de cada especie en los meses de muestreo (T1-T4), utilizando el índice de similaridad de Bray \& Curtis (1957). Todos los análisis se llevaron a cabo con el programa PRIMER-E v6 (Clarke \& Gorley 2006).

\section{Resultados}

Se identificaron 24 especies de algas rojas (Tabla 2), recolectándose 288 poliquetos pertenecientes a 19 especies de 7 familias. La familia Nereididae fue la mejor representada con 7 especies, seguida de Eunicidae con 5 y Syllidae con 3 especies. La especie más abundante fue Platynereis dumerilii Audouin y Milne Edwards, 1834 (Nereididae) con 188 individuos, seguida de Pseudonereis gallapagensis Kinberg, 1865 (Nereididae) con 23 individuos y Syllis corallicola Verrill, 1900 (Syllidae) con 12 individuos. También se reportaron los poliquetos sedentarios Hydroides sanctaecrusis Kroeyer in Mörch, 1863 (Serpulidae) con 4 individuos y Pista palmata Verrill, 1873 (Terebellidae) con 5 individuos (Tabla 3).

\begin{tabular}{|c|c|c|c|c|c|c|c|c|c|c|}
\hline \multirow{7}{*}{$\begin{array}{l}\text { Tabla 3. Listado de especies y } \\
\text { abundancia de poliquetos en } \\
\text { algas rojas intermareales de } \\
\text { Córdoba / List of species and } \\
\text { abundance of Polychaetes } \\
\text { from intertidal red algae of } \\
\text { Córdoba }\end{array}$} & \multirow{2}{*}{ Familia } & \multirow{2}{*}{ Especie } & \multicolumn{3}{|c|}{ Ecorregión Morrosquillo } & \multicolumn{4}{|c|}{ Ecorregión Darién } & \multirow{2}{*}{ Total } \\
\hline & & & Est.1 & Est. 2 & Est. 3 & Est. 4 & Est. 5 & Est.6 & Est.7 & \\
\hline & \multirow[t]{5}{*}{ Eunicidae } & Eunice cariboea Grube, 1856 & 7 & 1 & 0 & 0 & 0 & 0 & 0 & 8 \\
\hline & & Eunice filamentosa Grube, 1856 & 3 & 3 & 0 & 0 & 0 & 0 & 0 & 6 \\
\hline & & Eunice antennata (Savigny, 1820) & 0 & 1 & 3 & 0 & 0 & 0 & 0 & 4 \\
\hline & & Eunice websteri Fauchald, 1969 & 0 & 0 & 0 & 9 & 0 & 0 & 0 & 9 \\
\hline & & Marphysa regalis Verrill, 1900 & 1 & 0 & 1 & 0 & 0 & 0 & 0 & 2 \\
\hline & \multirow[t]{7}{*}{ Nereididae } & Laeonereis culveri (Webster, 1879) & 2 & 2 & 0 & 0 & 0 & 0 & 0 & 4 \\
\hline & & Platynereis dumerilii (Audouin \& Milne Edwards, 1834) & 10 & 22 & 13 & 20 & 41 & 24 & 58 & 188 \\
\hline & & Perinereis anderssoni Kinberg, 1866 & 0 & 0 & 5 & 0 & 0 & 0 & 0 & 5 \\
\hline & & Nereis pelagica occidentalis Hartman, 1945 & 0 & 0 & 0 & 0 & 0 & 2 & 0 & 2 \\
\hline & & Websterinereis tridentata (Webster, 1880) & 0 & 0 & 0 & 0 & 1 & 0 & 5 & 6 \\
\hline & & Pseudonereis gallapagensis Kinberg, 1865 & 0 & 0 & 0 & 9 & 7 & 4 & 3 & 23 \\
\hline & & Alitta succinea (Leuckart, 1847) & 0 & 0 & 0 & 0 & 0 & 0 & 3 & 3 \\
\hline & \multirow[t]{3}{*}{ Syllidae } & Syllis corallicola Verrill, 1900 & 2 & 2 & 2 & 5 & 0 & 1 & 0 & 12 \\
\hline & & Syllis cornuta Rathke, 1843 & 0 & 0 & 0 & 0 & 1 & 0 & 0 & 1 \\
\hline & & Myrianida sp. Milne Edwards, 1845 & 0 & 0 & 0 & 0 & 0 & 0 & 2 & 2 \\
\hline & Polynoidae & Lepidonofus variabilis Webster, 1879 & 0 & 3 & 0 & 0 & 0 & 0 & 0 & 3 \\
\hline & Serpulidae & Hydroides sanctaecrusis Kroeyer in Mörch, 1863 & 2 & 1 & 1 & 0 & 0 & 0 & 0 & 4 \\
\hline & Sabellariidae & Sabellaria floridensis Hartman, 1944 & 1 & 0 & 0 & 0 & 0 & 0 & 0 & 1 \\
\hline & Terebellidae & Pista palmata (Verrill, 1873) & 0 & 0 & 0 & 5 & 0 & 0 & 0 & 5 \\
\hline & \multicolumn{2}{|c|}{ Total de especies } & 8 & 8 & 6 & 5 & 4 & 4 & 5 & 19 \\
\hline & \multicolumn{2}{|c|}{ Total de individuos } & 28 & 35 & 25 & 48 & 50 & 31 & 71 & 288 \\
\hline
\end{tabular}


La abundancia total más alta de poliquetos se registró en diciembre (T2) y marzo (T3) con 79 y 103 individuos, existiendo sin embargo, una disminución en el porcentaje de cobertura algal durante estos meses de 71,1 a $58,7 \%$. La menor abundancia total ocurrió en septiembre (T1) y junio (T4) con 47 y 59 individuos, con un porcentaje algal de 48,9 y 70,4\%, respectivamente (Fig. 2). Con relación a las estaciones de muestreo, la mayor abundancia total de poliquetos se obtuvo en T2E5 con 30 individuos y la menor en T1E3 y T4E5 con un individuo (Fig. 2).

Al analizar la variación temporal de los poliquetos más representativos asociados a las algas rojas intermareales de Córdoba (Fig. 3), se encontró que 5 especies alcanzaron el $80,9 \%$ de la abundancia total; $P$. dumerilii presentó la abundancia más alta del total registrado durante el estudio, presentando los picos máximos en E5T2 (29 individuos, 15,4\%), E2T3 (19 individuos, 10,1\%) y E7T4 (26 individuos, 13,8\%); S. corallicola registró el máximo pico de abundancia en E4T2 (4 individuos, 33,3\%), mientras que $P$. gallapagensis se recolectó exclusivamente en el Darién cordobés con la abundancia más alta en E5T1 (4 individuos, 17,4\%) y E4T3 (5 individuos, 21,7\%). Además, se observó un patrón de distribución temporal de algunas especies; $H$. sanctaecrusis se registró en junio (T4) en E1, E2, y E3, mientras que Eunice filamentosa Grube, 1856 (Eunicidae) se recolectó en diciembre (T2) en E1 y E2.

Los poliquetos estuvieron representados por 7 familias, siendo Nereididae la más abundante durante todo el estudio (80,2\%), seguida de Eunicidae (10,1\%) y Syllidae $(5,2 \%)$. El resto de las familias presentaron una abundancia menor al 2\% (Fig. 4a). Por otro lado, según la procedencia de poliquetos en los frondes algales, los mayores valores de asociación (Fig. 4b) corresponden a las algas rojas de las familias Rhodomelaceae $(42,3 \%)$ y Gracilariaceae (26,9\%).

Se identificaron 5 grupos (A-E) de acuerdo con lo establecido con el UPGMA (Fig. 5a). El grupo A presenta $58 \%$ de similitud, corresponde al muestreo realizado en septiembre (T1) en las estaciones E1 y E2; El B con 43\% de similitud y el C con $41 \%$ corresponden a los 4 muestreos (T1-T4) en todas las estaciones del Darién cordobés, excepto E3; el grupo D con 51\% de similitud, pertenece al muestreo realizado en junio (T4) en las estaciones E1 y E2; y el grupo E con $46 \%$ de similitud, corresponde a los muestreos de diciembre (T2) y marzo (T3) en las estaciones E1 y E2.

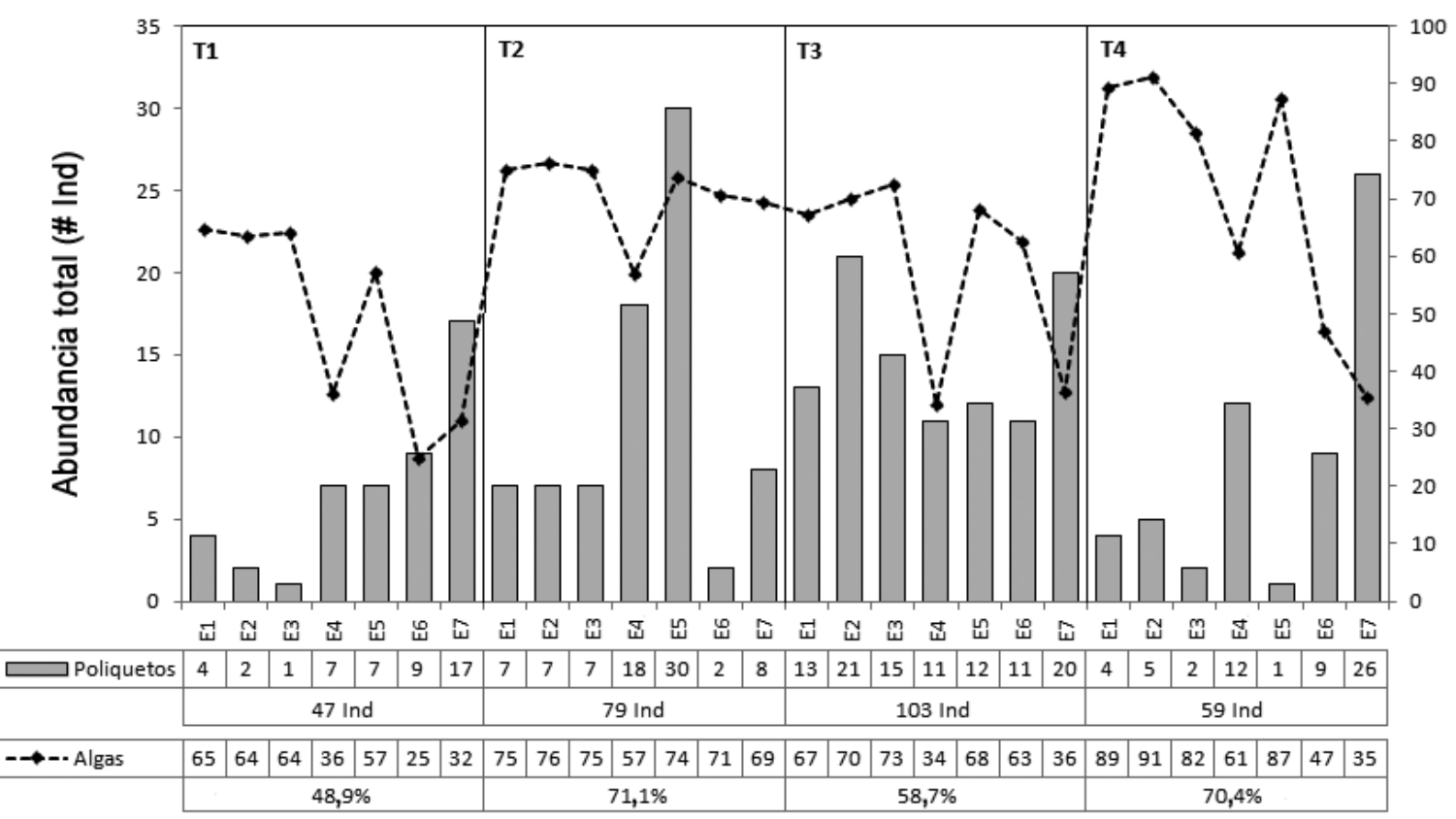

Figura 2. Abundancia total de poliquetos y su relación con la cobertura algal en los meses de muestreo (T1-T4) / Total abundance of polychaetes and its relation to algal coverage in the sampling months (T1-T4) 


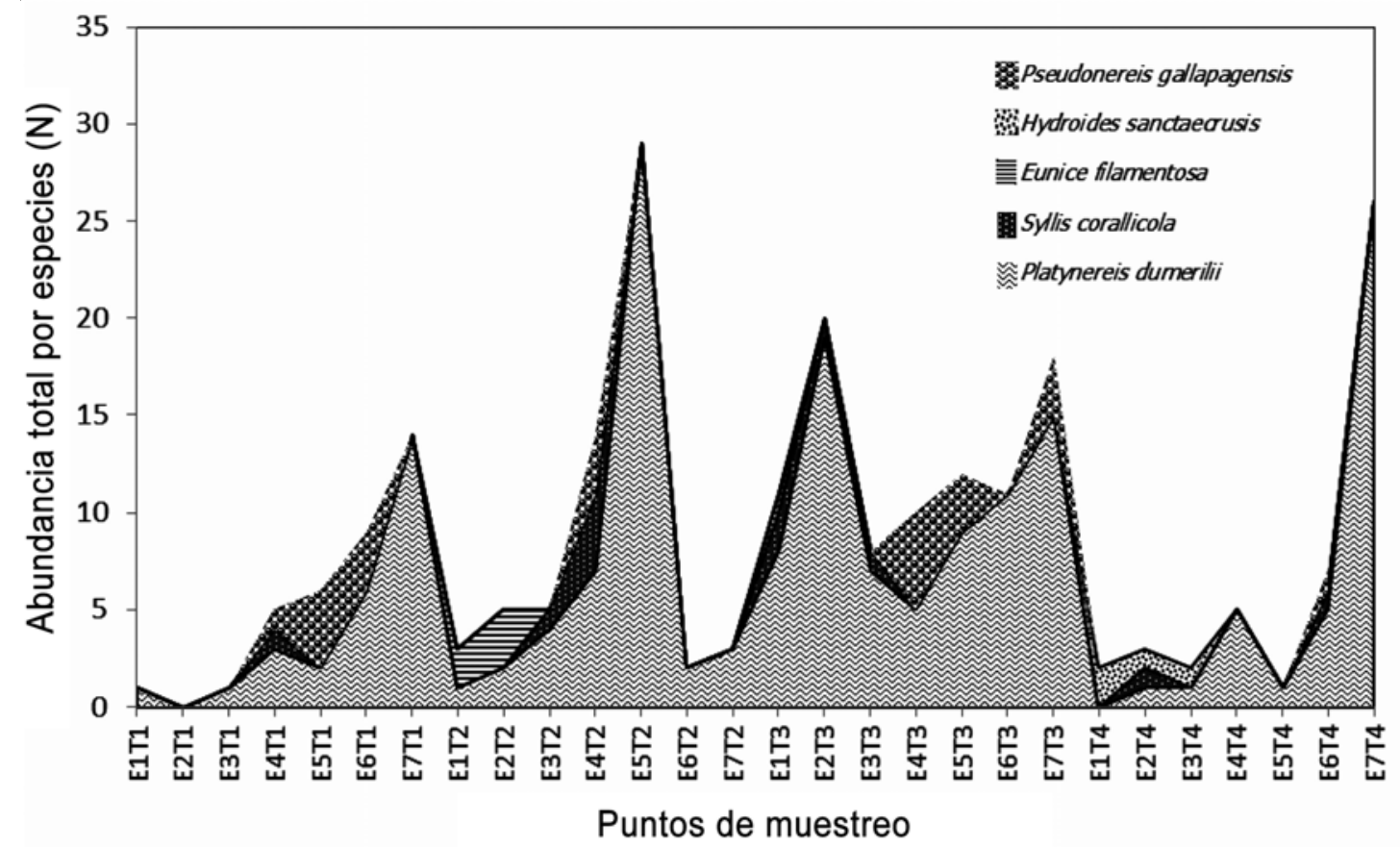

Figura 3. Comportamiento de los poliquetos dominantes asociados a las algas rojas intermareales de Córdoba / Abundance patterns of dominant polychaetes associated with intertidal red algae of Córdoba
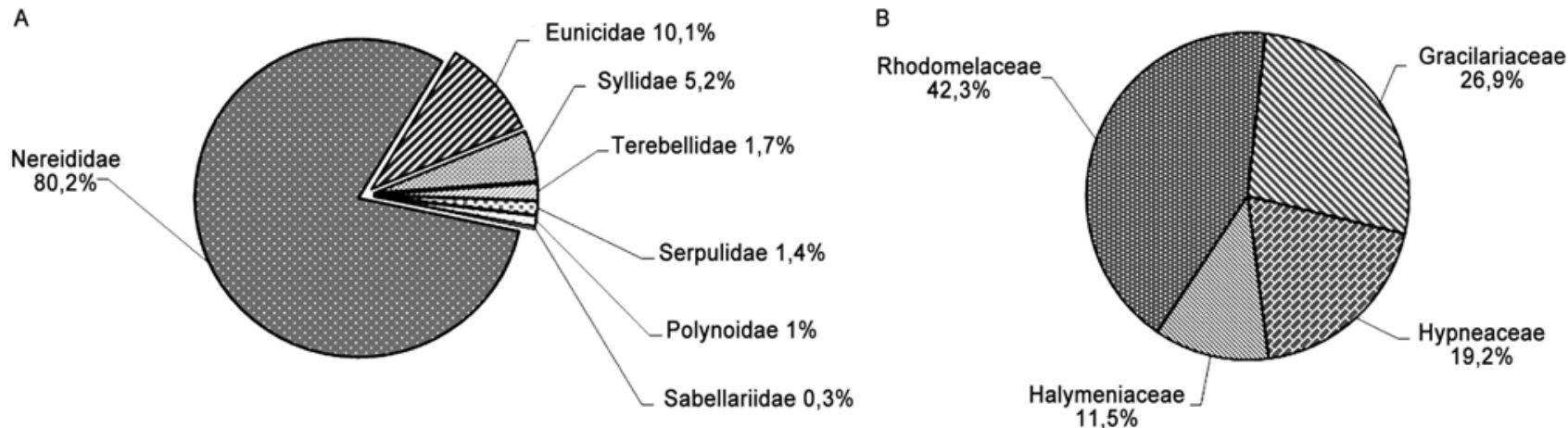

Figura 4. A) Abundancia relativa total por familias de poliquetos durante el periodo de estudio. B) Procedencia algal expresado en porcentaje del número de poliquetos / A) Total relative abundance of polychaete families during the study period. B) Red algal origin as a percentage of number of polychaetes 

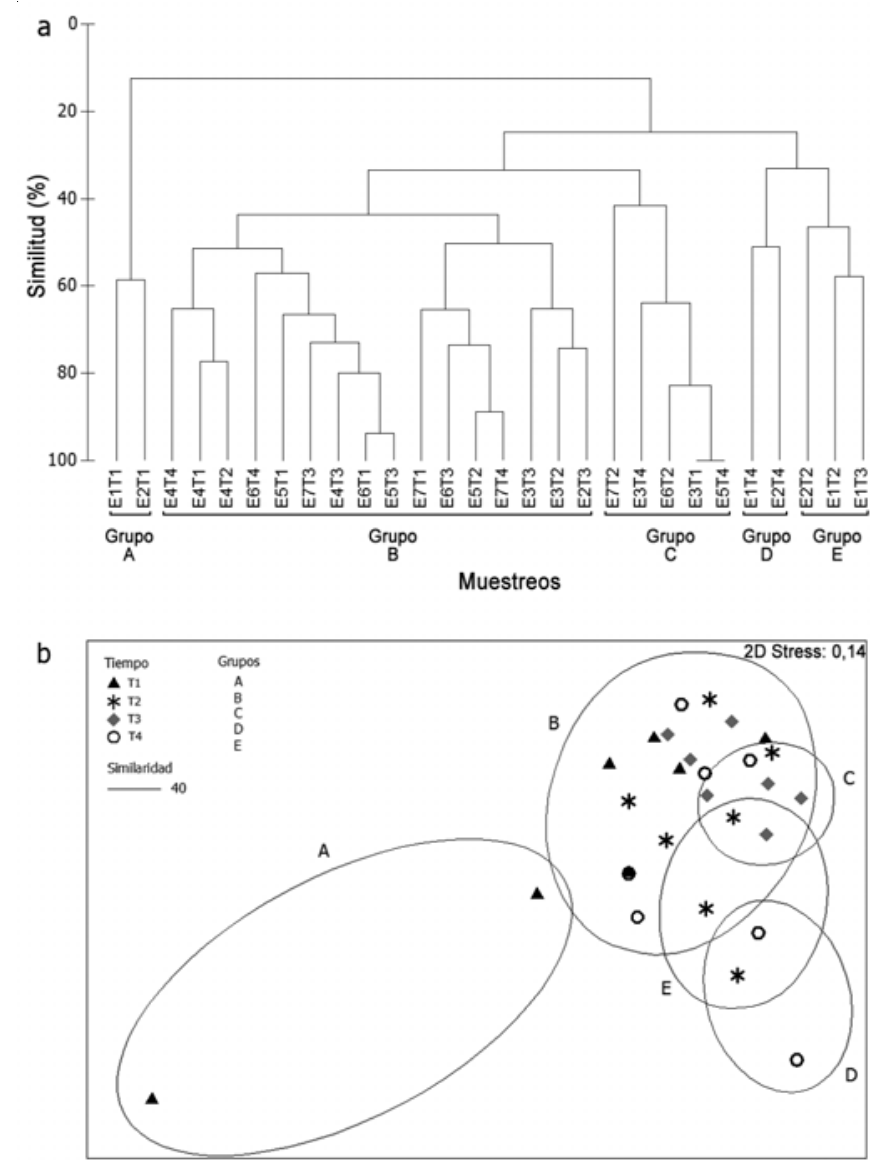

Figura 5. a) Dendrograma (UPGMA) y b) escalamiento no métrico multidimensional (NMDS) basados en la matriz de similitud de Bray-Curtis, utilizando la abundancia total de los poliquetos en algas rojas intermareales de Córdoba / a) Cluster (UPGMA) and b) Nonmetric multidimensional scaling (MDS) based on a Bray-Curtis similarity matrix using the total abundance of polychaetes from intertidal red algae of Córdoba

En el MNDS, se observa a los grupos B, C, D y E próximos entre sí, con relación a los meses de muestreo (Fig. 5b). El análisis también muestra a las estaciones E1 y E2 con una separación amplia en T1 (grupo A), a las estaciones del Darién cordobés determinadas desde T1 hasta T4 (grupos B y C) y a las estaciones E1 y E2 representadas por T4 (grupo D) y T2 (grupo E).

Con la prueba ANOSIM, no se registró una agrupación significativamente diferente de la otra $(\mathrm{R}=0,09, P=0,52)$, debido a que $P$. dumerilii mostró el porcentaje más alto de contribución (> 77\%) en cada agrupación, lo que indica que fue la especie más importante en términos de abundancia, en todos los meses muestreados (Tabla 4). Sin embargo, el promedio de similaridad con respecto a la combinación de los meses muestreados (T1-T4), responde a la presencia de esta especie asociada a los poliquetos errantes $P$. gallapagensis y $S$. corallicola y sedentarios H. sanctaecrusis y Pista palmata Verrill, 1873 (Terebellidae) (Tabla 5).
Tabla 4. Análisis de similitud porcentual de la abundancia de las especies de poliquetos con base a los meses muestreados (T1-T4) / Similarity Percentage Analysis of polychaetes species abundance based in sampling months (T1-T4)

\begin{tabular}{|c|c|c|c|c|c|}
\hline & Especie & $\begin{array}{c}\text { Abundancia } \\
\text { promedio }\end{array}$ & $\begin{array}{c}\text { Similaridad } \\
\text { promedio }\end{array}$ & $\begin{array}{c}\text { Contribución } \\
\text { (\%) }\end{array}$ & $\begin{array}{c}\text { Acumulado } \\
\text { (\%) }\end{array}$ \\
\hline \multirow[t]{3}{*}{$\mathrm{T} 1$} & & & 29,09 & & \\
\hline & P. dumerilii & 1,62 & 22,48 & 77,29 & 77,29 \\
\hline & P. gallapagensis & 0,68 & 3,82 & 13,13 & 90,41 \\
\hline \multirow[t]{2}{*}{$\mathrm{T} 2$} & & & 33,97 & & \\
\hline & P. dumerilii & 2,23 & 30,96 & 91,13 & 91,13 \\
\hline \multirow[t]{3}{*}{$\mathrm{T} 3$} & & & 54,61 & & \\
\hline & P. dumerilii & 3,18 & 47,55 & 87,06 & 87,06 \\
\hline & P. gallapagensis & 0,81 & 4,34 & 7,95 & 95,01 \\
\hline \multirow[t]{3}{*}{$\mathrm{T} 4$} & & & 29,43 & & \\
\hline & P. dumerilii & 1,80 & 22,77 & 77,37 & 77,37 \\
\hline & P. gallapagensis & 0,49 & 4,55 & 15,46 & 92,83 \\
\hline
\end{tabular}

TI; septiembre, T2; diciembre, T3; marzo, T4; junio 
Tabla 5. Análisis de similitud porcentual de la abundancia de las especies de poliquetos con base en la combinación de los meses de muestreo (T1-T4) / Similarity Percentage Analysis of the polychaetes species abundance based on the combination of the sampling months (T1-T4)

\begin{tabular}{|c|c|c|c|c|c|}
\hline $\begin{array}{c}\text { Combinación } \\
\text { T1-T4 }\end{array}$ & \multicolumn{2}{|c|}{$\begin{array}{l}\text { Abundancia } \\
\text { promedio }\end{array}$} & $\begin{array}{l}\text { Similaridad } \\
\text { promedio }\end{array}$ & $\begin{array}{c}\text { Contribución } \\
(\%)\end{array}$ & $\begin{array}{c}\text { Acumulado } \\
(\%)\end{array}$ \\
\hline $\mathrm{T} 1 \& \mathrm{~T} 2$ & & & 68,88 & & \\
\hline P. dumerilii & 1,62 & 2,23 & 16,61 & 24,11 & 24,11 \\
\hline P. gallapagensis & 0,68 & 0,25 & 8,10 & 11,75 & 35,87 \\
\hline S. corallicola & 0,14 & 0,43 & 4,83 & 7,01 & 44,88 \\
\hline E. websteri & 0,14 & 0,25 & 3,08 & 4,47 & 47,35 \\
\hline P. palmata & 0,14 & 0,14 & 2,25 & 3,26 & 50,61 \\
\hline $\mathrm{T} 1 \& \mathrm{~T} 3$ & & & 62,73 & & \\
\hline P. dumerilii & 1,62 & 3,18 & 20,96 & 33,41 & 33,41 \\
\hline P. gallapagensis & 0,68 & 0,81 & 10,18 & 16,23 & 49,64 \\
\hline S. corallicola & 0,14 & 0,49 & 5,01 & 7,99 & 57,63 \\
\hline $\mathrm{T} 2 \& \mathrm{~T} 3$ & & & 58,57 & & \\
\hline P. dumerilii & 2,23 & 3,18 & 15,92 & 27,18 & 27,18 \\
\hline P. gallapagensis & 0,25 & 0,81 & 8,23 & 14,04 & 41,23 \\
\hline S. corallicola & 0,43 & 0,49 & 5,62 & 9,60 & 50,83 \\
\hline P. palmata & 0,14 & 0,14 & 2,21 & 3,60 & 54,43 \\
\hline $\mathrm{T} 1$ y $\mathrm{T} 4$ & & & 71,80 & & \\
\hline P. dumerilii & 1,62 & 1,80 & 19,15 & 26,67 & 26,67 \\
\hline P. gallapagensis & 0,68 & 0,14 & 8,80 & 12,25 & 38,93 \\
\hline H. sanctaecrusis & 0,00 & 0,49 & 7,90 & 11,00 & 49,92 \\
\hline P. palmata & 0,14 & 0,20 & 3,37 & 4,69 & 54,61 \\
\hline $\mathrm{T} 2$ y $\mathrm{T} 4$ & & & 67,49 & & \\
\hline P. dumerilii & 2,23 & 1,80 & 18,03 & 26,71 & 26,71 \\
\hline H. sanctaecrusis & 0,00 & 0,49 & 6,59 & 9,77 & 36,48 \\
\hline E. cariboea & 0,25 & 0,34 & 6,04 & 8,95 & 45,43 \\
\hline S. corallicola & 0,43 & 0,29 & 5,54 & 8,22 & 53,65 \\
\hline $\mathrm{T} 3$ y $\mathrm{T} 4$ & & & 65,34 & & \\
\hline P. dumerilii & 3,18 & 1,80 & 21,92 & 33,55 & 33,55 \\
\hline P. gallapagensis & 0,81 & 0,14 & 9,05 & 13,86 & 47,40 \\
\hline H. sanctaecrusis & 0,00 & 0,49 & 5,59 & 8,55 & 55,95 \\
\hline
\end{tabular}

T1; septiembre, T2; diciembre, T3; marzo, T4; junio

\section{Discusión}

Las algas rojas, en sus formas frondosas, exhibieron una dominancia y permanencia temporal en el intermareal rocoso, coincidiendo con lo reportado por QuirósRodríguez et al. (2010). Esta tendencia de cobertura algal permanente durante todo el año, ofrece un área mayor para el asentamiento de poliquetos errantes, tubícolas y excavadores. Los numerosos hábitat que originan los frondes algales para el asentamiento y desarrollo de poliquetos, ya fue estudiado por Adami \& Gordillo (1999), Ríos et al. (2007) y García-Ríos et al. (2008), quienes sostienen que las algas rojas y pardas ofrecen a los organismos que viven en sus frondes un microhábitat protegido del impacto del movimiento del agua, creando condiciones más homogéneas que las del hábitat donde crece el alga.

La alta sensibilidad de estas algas a la intensidad solar, puede ser de algún modo reducida por la forma de crecimiento tan compacto, y en donde las múltiples ramificaciones de los frondes les permite retener por más tiempo la humedad en el intermareal, evitando el deterioro en su estructura vegetativa (Johansen 1974). Esta característica según Taylor (1975) y Stewart (1989), les confiere una gran capacidad de concentrar sedimentos, favoreciendo el desarrollo de una comunidad de poliquetos característica de sustratos de fondos blandos y rocosos.

El número de especies de poliquetos registrado en estudios con macroalgas es similar al obtenido en la presente investigación; así, Villouta \& Santelices (1984) registraron 9 especies asociadas a Lessonia nigrescens (Phaeophyta, Laminariales) a lo largo de Chile norte y central; López \& Stotz (1997) identificaron 15 especies asociadas a Corallina officinalis (Rodophyta, Corallinales) en el área costera de Palo Colorado, Chile; Adami \& Gordillo (1999) registraron 10 especies en Macrocystis pyrifera (Phaeophyta, Laminariales) en el Canal del Beagle, Tierra del Fuego, Argentina. Ríos et al. (2007) en 2 sectores del Estrecho de Magallanes, Chile, registraron 43 especies de poliquetos asociados a $M$. pyrifera, destacándose en términos de abundancia Platynereis australis Schmarda, 1861, Hermadion magalhaensis Kinberg, 1855 y Polycirrus sp. Grube, 1850. Según los autores, la heterogeneidad en las condiciones ambientales de la región de Magallanes, sugiere una mayor riqueza y dinámica de las poblacionales de poliquetos asociados con $M$. pyrifera.

El registro de 19 especies en esta región (16 poliquetos errantes y 3 sedentarios) evidencia la capacidad de las algas rojas intermareales en constituirse verdaderas islas ecológicas, en las cuales aumenta la riqueza de poliquetos en función al aumento en cobertura de los frondes algales (Kuris et al. 1980, De León González et al. 1993). La familia Nereididae fue el grupo dominante en los frondes algales con el $80,2 \%$ de la abundancia total, similar a lo descrito en Chile sobre parches de Pyura chilensis Molina, 1782 (Zamorano \& Moreno 1975), en el cirripedio Austromegabalanus psittacus Molina, 1782 (Hernández et al. 2001) y sobre agregaciones de Phragmatopoma 
moerchi Kinberg, 1867 (Sepúlveda et al. 2003) y en Perú sobre comunidades de mitílidos (Paredes \& Tarazona 1980). La escasa presencia de sabelaridos y terebélidos con $0,35 \%$ y $1,74 \%$ de la abundancia total, fue concordante con lo reportado en Chile sobre algas rojas articuladas (López \& Stotz 1997) y frondes de M. pyrifera (Adami \& Gordillo 1999).

El hecho que Platynereis dumerilii registre un rango de distribución amplio y dominancia en el intermareal rocoso de Córdoba, es concordante con el hecho de que ha sido descrita como habitante de sustratos duros y generalmente catalogada como una especie herbívora (San Martín \& Bone 2001), lo cual favorece su presencia en los frondes de algas rojas desde las cuales se puede alimentar. Por otra parte, Salazar-Vallejo \& Jiménez-Cueto (1996-1997) la caracterizan como un habitante permanente de las macroalgas, en donde cumple todo su ciclo de vida. Esto último determina que sea la especie de mayor abundancia durante todo el estudio.

Pseudonereis gallapagensis ha sido igualmente registrada en algas, y muy común en áreas tropicales (Díaz \& Liñero-Arana 2003), su distribución parece estar relacionada con una mayor influencia de aguas continentales y sedimentos, lo que aparentemente es determinante en el asentamiento de esta especie en la ecorregión Darién (E4-E7). Según Salazar-Vallejo \& Jiménez-Cueto (1996-1997) han registrado la especie en fondos mixtos con algas rojas, desde la zona litoral hasta $10 \mathrm{~m}$ de profundidad; esta especie fue registrada por primera vez para el Caribe colombiano por Dueñas-Ramírez \& Quirós-Rodríguez (2012). Syllis corallicola ha sido reportada en algas, esponjas, interior de corales muertos, fanerógamas marinas y en arena gruesa; además es catalogada como una especie carnívora y omnívora con faringe eversible que utiliza los frondes de algas rojas como zonas de alimentación (Fauchald 1977), lo cual fundamenta su abundancia en todos las estaciones del intermareal rocoso de Córdoba.

El asentamiento de Hydroides sanctaecrusis y Sabellaria floridensis en los frondes de algas rojas, probablemente se debe a que éstos se constituyen un sustrato adecuado para construcción de tubos de carbonato de calcio y arena. Por otro lado, la acumulación de sedimentos sobre estos frondes, permite a Pista palmata construir sus galerías a pequeña escala dentro de los sedimentos. Sin embargo, el número de especies y de individuos de serpúlidos, sabeláridos y terebélidos, fue inferior a lo registrado por Sepúlveda et al. (2003) y Liñero-
Arana \& Díaz-Díaz (2006), debido posiblemente a que las algas rojas desarrollan diferentes adaptaciones para dificultar o impedir el asentamiento de epibiontes, entre las que se encuentran la producción de mucus y emisión de iones libres que impide la fijación (Wahl 1989) y exudado de metabolitos secundarios y otros compuestos bioactivos que impiden el asentamiento de organismos (Slattery et al. 1995).

En un plano bidimensional MNDS, los grupos B, C, D y E se encuentran muy próximos entre sí, con relación a los meses de muestreo; por lo cual, aunque existe un patrón de distribución temporal que se refleja en E1 y E2, en general para el intermareal rocoso de Córdoba, esta distribución presenta limites difusos, que puede atribuirse a la abundancia de especies como $P$. dumerilii y $P$. gallapagensis, que al tolerar amplios rangos ambientales y establecerse en diferentes ecosistemas (Díaz \& LiñeroArana 2003), presentan una distribución más amplia, lo que se muestra en los altos porcentajes de similitud (por agrupación) arrojados por el análisis de clasificación UPGMA. Según García-Ríos et al. (2008) porcentajes altos de similitud ( $>50 \%$ ) es característico de comunidades poco diversas, con alta dominancia y baja variabilidad en la frecuencia de especies entre localidades, lo que se ajusta con el presente estudio.

La estructura vegetativa y las variadas formas de crecimiento de los frondes de algas rojas intermareales, resultan ser un importante hábitat para el asentamiento de especies de poliquetos ya sea de sustratos de fondos blandos y rocosos, los cuales pueden ser utilizados en programas de monitoreo, dirigidos en la conservación de la diversidad marina local.

\section{Agradecimientos}

Los autores agradecen a los biólogos Emilio Isaza, Oscar Pérez, Mauricio Díaz, Ana Carolina Gonzales, y Diana Herrera, por el apoyo dado en la realización del estudio. A la División de Investigación de la Universidad de Córdoba por el financiamiento recibido a través de los Proyectos; Composición y cambios estacionales de las poblaciones de crustáceos decápodos del departamento de Córdoba (FCB 0705) y distribución espacio temporal de las comunidades macroalgales asociadas al litoral rocoso del departamento de Córdoba, Caribe colombiano (FCB 0404). Contribución $\mathrm{N}^{\circ} 380$ del Centro de Estudios en Ciencias del Mar, CECIMAR de la Universidad Nacional de Colombia, Sede Caribe. 


\section{LITERATURA CITADA}

Adami ML \& S Gordillo. 1999. Structure and dynamics of the biota associated with Macrocystis pyrifera (Phaeophyta) from the Beagle Channel, Tierra del Fuego. Scientia Marina 63 (Suppl. 1): 183-191.

Alós C, A Campoy \& F Pereira. 1982. Contribución al estudio de los anélidos poliquetos endosimbiontes de esponjas. Actas II Simposio Ibérico de Estudios del Bentos Marino 3: 139-157.

Ardila N, GR Navas \& J Reyes. 2002. Libro rojo de invertebrados marinos de Colombia, 180 pp. Serie Libros Rojos de Especies Amenazadas de Colombia, INVEMAR, Ministerio de Medio Ambiente, Bogotá.

Bates CR. 2009. Host taxonomic relatedness and functionalgroup affiliation as predictors of seaweed-invertebrate epifauna associations. Marine Ecology Progress Series 387: 125-136.

Bates CR \& R DeWreede. 2007. Do changes in seaweed biodiversity influence associated invertebrate epifauna? Journal of Experimental Marine Biology and Ecology 344: 206-214.

Bula-Mayer G. 1998. Estado actual de la taxonomía de las macroalgas marinas de Colombia. Boletín Ecotrópica 33: 113.

Bray JR \& JT Curtis. 1957. An ordination of the upland forest communities of southern Wisconsin. Ecological Monographs 27: 325-349.

Cañete J, G Leighton \& E Soto. 2000. Proposición de un índice de vigilancia ambiental basado en la variabilidad temporal de la abundancia de dos especies de poliquetos bentónicos de bahía Quintero, Chile. Revista de Biología Marina y Oceanografía 35: 185-194.

Cariceo Y, E Mutschke \& C Ríos. 2002. Ensambles de Isopoda (Crustacea) en discos de fijación del alga Macrocystis pyrifera (L.) C. Agardh (Phaeophyta) en el estrecho de Magallanes, Chile. Anales del Instituto de la Patagonia 30: 83-94.

Clarke KR \& RN Gorley. 2006. PRIMER-E v6: User Manual/ Tutorial, 96 pp. Plymouth.

De León-González JA, A Leija-Tristán \& S SalazarVallejo. 1993. Epifauna del ostión espinoso Spondylus princeps unicolor (Mollusca: Bivalvia) de Puerto Escondido, Golfo de California, México. Revista de Biología Tropical 41: 877-881.

Díaz 0 \& I Liñero-Arana. 2003. Poliquetos epibiontes de Pinctada imbricata Röding, 1758 (Bivalvia: Pteriidae) en el Golfo de Cariaco, Venezuela. Interciencia 28(5): 298-301.

Dueñas-Ramírez PR \& J Quirós-Rodriguez. 2012. Occurrence of Pseudonereis gallapagensis Kinberg, 1865 (Annelida: Polychaeta: Nereididae) in the Colombian Caribbean coast. Revista Colombiana de Ciencia Animal 4(2): 454-457.
Fauchald K. 1977. The Polychaeta worms. Definitions and keys to the Orders, Families and Genera. Natural History Museum of Los Angeles County, Science Series 28: 1-190.

García C \& G Díaz-Pulido. 2006. Dynamics of a macroalgal rocky intertidal community in the Colombian Caribbean. Boletín de Investigaciones Marinas y Costeras 35: 7-18.

García-Ríos C, F Soto-Santiago, R Colón-Rivera \& J Medina-Hernández. 2008. Gastrópodos asociados al alga calcárea Halimeda opuntia (Udoteaceae) en Puerto Rico. Revista de Biología Tropical 56(4): 1665-1675.

Hartmann-Schröder G. 1962. Zur Kenntnis des Eulitorals der chilenischen Pazifikküste und der argentinischen Küste Südpatagoniens unter besonderer Berücksichtigung der Polychaeten und Ostracoden. Tl.II. Die Polychaeten des Eulitorals. The Mitteilungen aus dem Hamburgischen Zoologischen Museum und Institut 60(Suppl.): 57-167.

Hartmann-Schröder G. 1965. Zur Kenntnis des Sublitorals der chilenischen Küste unter besonderer Berücksichtigung der Polychaeten und Ostracoden. Tl.II. Die Polychaeten des Sublitorals. The Mitteilungen aus dem Hamburgischen Zoologischen Museum und Institut 62(Suppl.): 59-305.

Hernández C, G Muñoz \& N Rozbaczylo. 2001. Poliquetos asociados con Austromegabalanus psittacus (Molina, 1782) (Crustacea: Cirripedia) en la Península Gualpén, Chile Central: Biodiversidad y efecto del tamaño del sustrato biológico. Revista de Biología Marina y Oceanografía 36(1): 99-108.

Höldridge L. 1978. Ecología basada en las zonas de vida, 216 pp. Instituto Interamericano de Ciencias Agrícolas, San José.

INVEMAR. 2000. Programa nacional de investigación en biodiversidad marina y costera. Instituto de Investigaciones Marinas y Costeras/FONADE/MMA. Santa Marta, Colombia 80 pp.

Jenkins G, G Walker-Smith \& P Hamer. 2002. Elements of habitat complexity that influence harpacticoid copepods associated with seagrass beds in a temperate bay. Oecologia 131: 598-605.

Johansen HW. 1974. Articulated coralline algae. Oceanography and Marine Biology: An Annual Review 12: 77-127.

Kelaher BP. 2002. Influence of physical characteristics of coralline turf on associated macrofaunal assemblages. Marine Ecology Progress Series 232: 141-148.

Kelaher BP \& J Castilla. 2005. Habitat characteristics influence macrofaunal communities in coralline turf more than mesoscale coastal upwelling on the coast of Northern Chile. Estuarine Coastal and Shelf Science 63: 155-165.

Kuris AM, AR Blaustein \& JJ Alió. 1980. Hosts as islands. The American Naturalist 116: 570-586.

Liñero-Arana I \& O Díaz-Díaz. 2006. Poliquetos (Annelida: Polychaeta) epibiontes de Spondylus americanus (Bivalvia: Spondylidae) en el Parque Nacional Mochima, Venezuela. Revista de Biología Tropical 54(3): 765-772. 
Littler DS \& MM Littler. 2000. Caribbean Reef Plants: An identification guide to the reef plants of the Caribbean, Bahamas, Florida, and Gulf of Mexico, 542 pp. Offshore Graphics, Washington.

López CA \& WB Stotz. 1997. Descripción de la fauna asociada a Corallina officinalis L. en el intermareal rocoso de la costa de 'Palo Colorado' (Los Vilos, IV-Región, Chile). Revista de Biología Marina y Oceanografía 32(1): 17-35.

Paredes C \& J Tarazona. 1980. Las comunidades de mitílidos del mediolitoral rocoso del departamento de Lima. Revista Peruana de Biología 2(1): 59-71.

Quirós-Rodríguez J, J Arias \& R Ruíz. 2010. Estructura de las comunidades macroalgales asociadas al litoral rocoso del departamento de Córdoba, Colombia. Caldasia 32(2): 339-354.

Ríos C \& E Mutschke. 2009. Aporte al conocimiento de Macrocystis pyrifera: Revisión bibliográficas sobre los 'Huirales' distribuidos en la región de Magallanes. Anales del Instituto de la Patagonia 37(1): 97-102.

Ríos C, WE Arntz, D Gerdes, E Mutschke \& A Montiel. 2007. Spatial and temporal variability of the benthic assemblages associated to the holdfasts of the kelp Macrocystis pyrifera in the Straits of Magellan, Chile. Polar Biology 31: 89-100.

Rozbaczylo N. 1980. Clave para el reconocimiento de familias de anélidos poliquetos del mar chileno. Studies on Neotropical Fauna and Environment 15(3-4): 167-196.

Salazar-Vallejo SI \& MS Jiménez-Cueto. 1996-1997. Neréididos (Polychaeta) del Caribe Mexicano con una clave para las especies del Gran Caribe. Revista de Biología Tropical 44(3)/45(1): 361-377.

San Martín G \& D Bone. 2001. Syllidae (Polychaeta) de praderas de Thalassia testudinum en el Parque Nacional Morrocoy (Venezuela). Revista de Biología Tropical 49: 609-620.

Schnetter R. 1980. Algas marinas nuevas para los litorales colombianos del mar Caribe. Caribbean Journal of Science 15: 121-125.

Seed R \& RJ O’Connor. 1981. Community organization in marine algal epifauna. Annual Review of Ecology and Systematic 12: 49-74.
Sepúlveda R, R Moreno \& F Carrasco. 2003. Diversidad de macroinvertebrados asociados a arrecifes de Phragmatopoma moerchi Kinberg, 1867 (Polychaeta: Sabellariidae) en el intermareal rocoso de Cocholgüe, Chile. Gayana 67(1): 45-54.

Slattery M, JB McClintock \& JN Heineb. 1995. Chemical defenses in Antarctic soft corals: evidence for antifouling compounds. Journal of Experimental Marine Biology and Ecology 190: 61-77.

Stewart JG. 1989. Establishment, persistence and dominance of Corallina (Rodophyta) in algal turf. Journal of Phycology 25: 436-446.

Taylor R. 1975. Marine algae of Great Swan Island. Atoll Research Bulletin 185: 6-10.

Unsworth R, S De Grave, J Jampa, D Smith \& J Bell. 2007. Faunal relationship with seagrass habitat structure: a case study using shrimp from the Indo-Pacific. Marine and Freshwater Research 58: 1008-1018.

Vásquez JA \& B Santelices. 1984. Comunidades de macroinvertebrados en discos adhesivos de Lessonia nigrescens Bory (Phaeophyta) en Chile central. Revista Chilena de Historia Natural 57: 131-154.

Vásquez JA \& JM Vega. 2005. Macroinvertebrados asociados a discos de adhesión de algas pardas: Biodiversidad de comunidades discretas como indicadoras de perturbaciones locales y de gran escala. En: Figueroa E (ed). Biodiversidad marina: Valoración, usos y perspectivas ¿Hacia dónde va Chile?, pp. 429-450. Editorial Universitaria, Santiago de Chile.

Villouta E \& B Santelices. 1984. Estructura de la comunidad submareal de Lessonia (Phaeophyta, Laminariales) en Chile norte y central. Revista Chilena de Historia Natural 57: 111-122.

Wahl M. 1989. Marine epibiosis. I. Fouling and antifouling: some basic aspects. Marine Ecology Progress Series 58: 175-189.

Winston JE \& N Eiseman. 1980. Bryozoan-algal associations in coastal and continental shelf waters of Eastern Florida. Florida Scientist 43(2): 65-74.

Zamorano JH \& CA Moreno. 1975. Comunidades bentónicas del sublitoral rocoso de bahía Corral: I. Área mínima de muestreo y descripción cuantitativa de la asociación de Pyura chilensis Molina. Medio Ambiente 1(1): 58-65.

Recibido 10 de octubre de 2012 y aceptado el 23 de febrero de 2013

Editor: Claudia Bustos 